INPLASY

PROTOCOL

To cite: Chen et al. Pre-/ probiotics improves postoperative adaptive immunity in colorectal cancer patients: a systematic review and meta-analysis. Inplasy protocol 202160011. doi: 10.37766/inplasy2021.6.0011

Received: 05 June 2021

Published: 05 June 2021

Corresponding author:

Renxu Lai

lairenxu@mail.sysu.edu.cn

Author Affiliation:

The Fifth Affiliated Hospital of

Sun Yat-sen University

Support: Not Applicable.

Review Stage at time of this submission: Preliminary searches.

Conflicts of interest:

None declared.

\section{Pre-/probiotics improves postoperative adaptive immunity in colorectal cancer patients: a systematic review and meta-analysis}

Chen, J1; Liang, H2; Lu, J3; He, Y4; Lai, R5

Review question / Objective: This meta-analysis aimed to evaluate the overall effect of pre-/probiotics on postoperative adaptive immunity in perioperative colorectal cancer patients. Condition being studied: Previous meta-analyses have suggested that oral pre-/probiotics improves clinical outcomes in patients with colorectal cancer during perioperative period. However, the effect of the pre-l probiotics on postoperative immunity was not included in those analyses.

Information sources: We searched electronic databases, including PubMed, the Cochrane Library, EMBASE, Ovid and China Biology Medicine Disc (http://www.sinomed.ac.cn/).

INPLASY registration number: This protocol was registered with the International Platform of Registered Systematic Review and Meta-Analysis Protocols (INPLASY) on 05 June 2021 and was last updated on 05 June 2021 (registration number INPLASY202160011).

\section{INTRODUCTION}

Review question / Objective: This metaanalysis aimed to evaluate the overall effect of pre-/probiotics on postoperative adaptive immunity in perioperative colorectal cancer patients.
Condition being studied: Previous metaanalyses have suggested that oral pre-/ probiotics improves clinical outcomes in patients with colorectal cancer during perioperative period. However, the effect of the pre-/probiotics on postoperative immunity was not included in those analyses. 


\section{METHODS}

Participant or population: Perioperative colorectal cancer patients.

Intervention: Use of enteral pre-/probiotics during perioperative period.

Comparator: No use of enteral pre-/ probiotics during perioperative period.

Study designs to be included: Randomized controlled trial.

Eligibility criteria: (1) study design: randomized controlled trial; (2) participants: perioperative colorectal cancer patients; (3) intervention versus control: pre-/probiotics use versus non-use; and (4) outcomes: levels of serum CD4+, CD8+, CD4+/CD8+, $\lg G, \lg A$, and $\lg M$ (7-12 days after surgery).

Information sources: We searched electronic databases, including PubMed, the Cochrane Library, EMBASE, Ovid and China Biology Medicine Disc (http:// www.sinomed.ac.cn/).

Main outcome(s): The outcomes included the levels of postoperative serum CD4+ Tcells, CD8+ T-cells, CD4+-to-CD8+ ratio, $\lg G$, IgA, and IgM (7-12 days after surgery).

Quality assessment / Risk of bias analysis: We used the Cochrane Collaboration tool to assess the risk of bias of the RCTs from the following 7 aspects: sequence generation, allocation concealment, blinding of participants and personnel, blinding of outcome assessors, incomplete outcome data, selective outcome reporting and other bias.

Strategy of data synthesis: We used mean difference (MD) and $95 \%$ confidence interval $(95 \% \mathrm{Cl})$ to estimate the pooled outcomes. Heterogeneity across studies was calculated by the $Q$ test and 12 statistic. If there was no heterogeneity, we used a fixed-effects model, otherwise, the random-effects model was used.

Subgroup analysis: The following subgroup analyses may be used: High-quality studies versus low-quality studies; Large sample studies versus small sample studies; Pre-/ probiotics use $\geq 5$ days vs Pre-/probiotics use $<5$ days.

Sensitivity analysis: We performed sensitivity analyses to assess the relative influence by excluding the studies one by one and changing the effects model.

Language: No language limits be imposed on the search.

Country(ies) involved: China.

Keywords: pre-/probiotics; colorectal cancer; adaptive immunity; perioperative period; meta-analysis.

Contributions of each author:

Author 1 - Jie Chen.

Email: 18270916863@163.com

Author 2 - Huamin Liang.

Email: 1124373189@qq.com

Author 3 - Jiaying Lu.

Email: ljyljymz@163.com

Author 4 - Yanping He.

Author 5 - Renxu Lai.

Email: lairenxu@mail.sysu.edu.cn 